\title{
Identification of Practices for Proper Implementation of Requirements in Global Software Development: A Systematic Literature Review Protocol
}

\author{
Atta Ur Rahman \\ Department of Computer \\ Science, COMSATS University \\ Islamabad, Islamabad \\ Pakistan
}

\author{
Muhammad Yaseen \\ Engineering Research \& IT \\ Services Provider (Pvt) Ltd \\ Peshawar, Pakistan
}

\author{
Zahid Ali \\ Engineering Research \& IT \\ Services Provider (Pvt) Ltd \\ Peshawar, Pakistan
}

\begin{abstract}
Effective requirement implementation leads to successful delivery of software. The requirement engineering (RE) is very difficult when implemented locally but the case is worst in global software development (GSD). There exist challenges such as 'Lack of effective communication', 'Organizational change', 'Lack of coordination and collaboration', 'Lack of knowledge-sharing and awareness' in GSD and to minimize the effect of these challenges success factors such as 'Support of collaborative tools', 'Global project management', 'Requirement Engineering modelling', and 'Proper negotiation and discussion' are suggested by many authors. To decrease the effect of challenges and to successfully implement success factors, practices and solutions are needed. The objective of this research is to find the practices needed to successfully implement RE process. Systematic Literature Review (SLR) is conducted for the identification of these practices
\end{abstract}

\section{Keywords}

Keywords: Practices/Solutions, Systematic literature review, Global software development, Requirement engineering.

\section{INTRODUCTION}

Requirement engineering (RE) is a systematic and proper way of collecting requirements from user by applying various techniques such as background study and interview [1]. RE is divided in some phases such as requirement elicitation in which we collect requirements from users, requirement analysis phase in which we examine user requirements using models, requirement specification phase in which we specify functional requirements of users and validation phase in which the collected user requirements are validated. The quality of implemented software is totally depended on proper requirement implementation. The more errors and bugs remain during $\mathrm{RE}$, the more is the chances of failure of the software and more it is expensive to fix it latterly on [2]. So $\mathrm{RE}$ needs more attention and much efforts are required. In GSD, RE is very difficult because there exists challenges such as culture difference, physical and geographical change, language and terminology difference, lack of face to face conversation and time zone difference [3][4]. To successfully implement RE, we should implement success factors in order to reduce the effect of challenges [5]. For every success factor we need practices. Practices are required for requirement elicitation, analysis, negotiation, specification phase and validation phase [6][7]. In our previous study, we have identified critical success factors for RE in GSD through SRL

[8]. The objective of this research work is to find all possible practices for implementing RE successfully.

\section{BACKGROUND STUDY}

Javed Iqbal [9] identified several RE practices in GSD. For requirement elicitation author identified practise like "collect requirements from multiple viewpoints", "reuse requirements from already developed similar systems", "identify stakeholders of the system and consulting them", "Recording requirement originating sources", "access system feasibility". He also identified practices like "define system boundaries", "use checklists for requirements analyses for Requirement analysis and negotiations". Further author identified practices like "model system environment", "Use structured methods for system modelling" for system modelling during RE. Practices for RE management like "define policies for requirement change management", "identify volatile requirements", "recording of the rejected requirements" are also identified by the author. Miguel Romero [10] discuss some of the practices for successful requirement elicitation such as the skills required for requirement elicitation in GSD are "English language skills", "virtual team skills", "computer mediated communication skills", "teamwork skills". Similarly knowledge and skills are required for requirement specification like "knowledge of the requirement attributes", "writing a draft of system definition document" etc. some practices for fundamental software requirements are also identified like "to know about different types of requirements and non-functional requirements also", "to analyse the requirements syntax", 'knowledge of system requirements specification', "knowledge of the specification knowledge". Jyoti M.Bhat [11] in his paper identified some practices to achieve the strategic success factors. "Develop stakeholder viewpoint", "build the team vision collaboratively", "use a human facilitator in integrated", "rich communication media during decision making" are some of the practices for shared goals. For shared culture some practices like "provide culture training to team members", "train team members on using communication technology", "share requirements specification templates", "establish technology accessibility and compatibility for all team" are identified.

For trust building factor the practices like "build team vision collaboratively", "get team together at the formation stage for a face to face kick-off session' are identified. Micheal Geisser [12] discuss some of the practices for requirement specification and quality of SRS like "correctness", "unambiguousness", “completeness", “consistency”, "prioritization and stability ranking", "verifiability", "modifiability", in his paper. 
Although from literature review, we identified some practices from the work of several authors but still we need SLR to identify from all possible literature best practices for the stated success factors discussed in section 3 below.
Previously we conducted SLR and identified success factors as shown in table 1 . The factors were analyzed on different research methods, time period, software company size and continents [8]. Finding these success factors are the base for our current research work.

Table 1: success factor identified through SLR

\begin{tabular}{|c|c|c|}
\hline S-no & Success factor & Occurrence \\
\hline 1 & Effective and strong communication system & $81 \%$ \\
\hline 2 & 3C (coordination, cooperation and collaboration) & $68 \%$ \\
\hline 3 & Using collaborative tools & $58 \%$ \\
\hline 4 & Knowledge Management and sharing & $53 \%$ \\
\hline 5 & Effective management & $51 \%$ \\
\hline 6 & RE modelling & $45 \%$ \\
\hline 7 & Proper discussion on requirements & $38 \%$ \\
\hline 8 & Software engineering process maturity & $30 \%$ \\
\hline 9 & Mutual Trust & $30 \%$ \\
\hline 10 & Requirement change management & $25 \%$ \\
\hline 11 & Training sessions & $17 \%$ \\
\hline 12 & Organizational proximity & $24 \%$ \\
\hline 13 & Use of new technologies & $5 \%$ \\
\hline 14 & Social networking & \\
\hline 15 & Infrastructure and organizational setup & 5 \\
\hline & & \\
\hline
\end{tabular}

\section{RESEARCH METHOD}

For the identification of practices for proper implementation of Critical Success Factors (CSFs), we used Systematic Literature Review (SLR) process. A similar approach has also been used by other researchers [13][14], we also studied these approaches. Figure 1 shows step by step design of research methodology. In first phase of SLR, research questions are finalized. In the second phase literature review will be conducted. The selection of relevant literature is selected on the bases of title and abstract in the third phase. In the fourth phase data are extracted from the relevant papers and also we synthesis these data into different categories. Finally, we classify these categories and identify practices for RE in GSD.

In order to identify RE practices for proper implementation of various CSFs in GSD, we have formulated the following research question (RQ1).

RQ1. What are the solutions/practices, as identified in the literature for proper implementation of success factors for RE in the context of GSD?
Research Question Identification

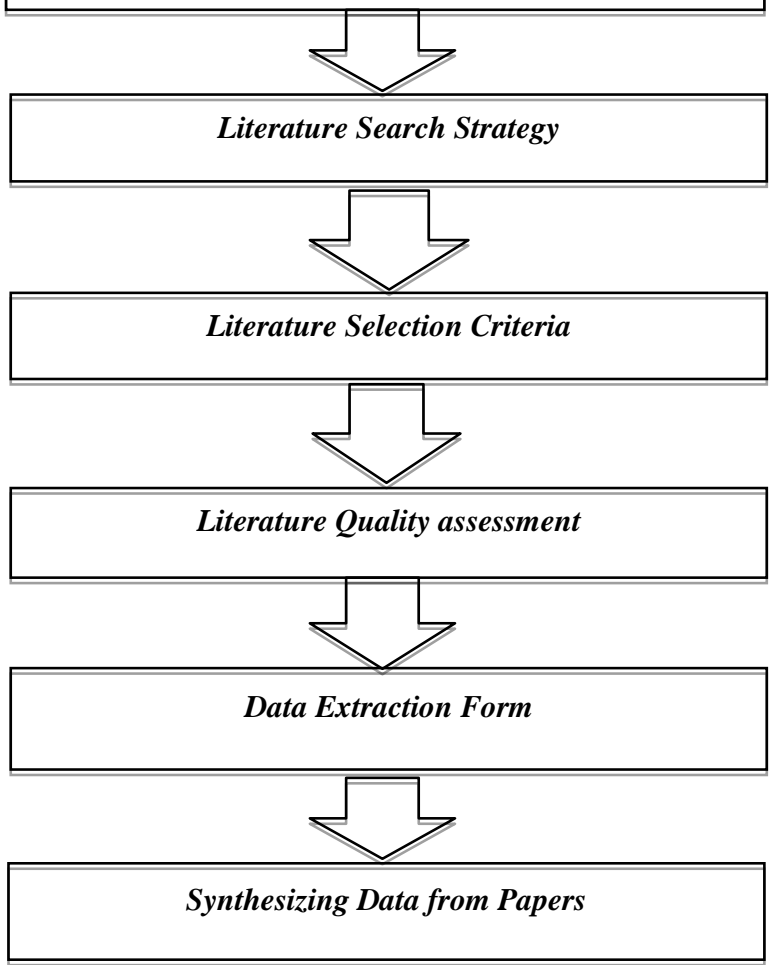

Fig.1 Development process for the SLR Protocol 


\subsection{SEARCH STRATEGY}

Search strategy include the following phases;

- $\quad$ Range of search ( time and space)

- Method for searching

- $\quad$ Electronic data sources used

- $\quad$ Strings for the search

- Validation of search

- Documentation of the search

- Management of search result

\subsubsection{Range of search (time and space)}

Refer to research questions we will search for all published literature with no bound and limit on any time (years).

\subsubsection{Method for searching}

A manual search was conducted for the determination of resources to be searched. We used the below resources for searching.

\subsubsection{Electronic data sources used}

The following digital resources are used for papers extraction

- Google scholar

- $\quad$ Science Direct

- $\quad$ Springer link

- Acm portal

- IEEE Xplore

\subsubsection{Strings for the search}

The following search string is used as a trial Search.

((Solutions OR practices OR "best practice" OR "lessons learned" OR Advice) AND ("Requirement Engineering" OR "Requirement Implementation" OR "Requirement Elicitation") AND ("Global Software development" OR "Distributed Software Development" OR "Software outsourcing")

\subsubsection{Documentation of the search}

Proper documentation of search results is necessary, and the following data will be listed:

- Database name

- Strategy for the search
- $\quad$ Phase of the search

- $\quad$ Search date

- No of publications found

- No of publication chosen

- Decision of introductory chosen

- Decision of final chosen

\subsection{Selection of Publication}

Selection process consist of following.

- Inclusion Criteria

- $\quad$ Removing Criteria

- Determination of Publication Quality

\subsubsection{Inclusion Criteria}

Entry criteria will be used to limit the number of papers which are retrieved by applying search strings and which are included for final data selection. The following are some inclusion criteria

- Papers written in English only are acceptable

- $\quad$ Papers which are related to RE in GSD only

- Papers which discuss the challenges only during requirement implementation in GSD.

- Studies that are related to RE only but they are fitted in GSD also.

\subsubsection{Exclusion criteria}

On the basis of removing criteria we decide which paper will be removed from the final list. The following are some exclusion criteria on base of which we will exclude papers from selected ones

- Studies which are not related to our Research questions

- $\quad$ Studies that do not discuss RE in GSD

- Studies that do not discuss challenges during RE in GSD.

- Papers belong to GSD but they don't discuss RE

- Papers that discuss RE but don't fit in GSD. 


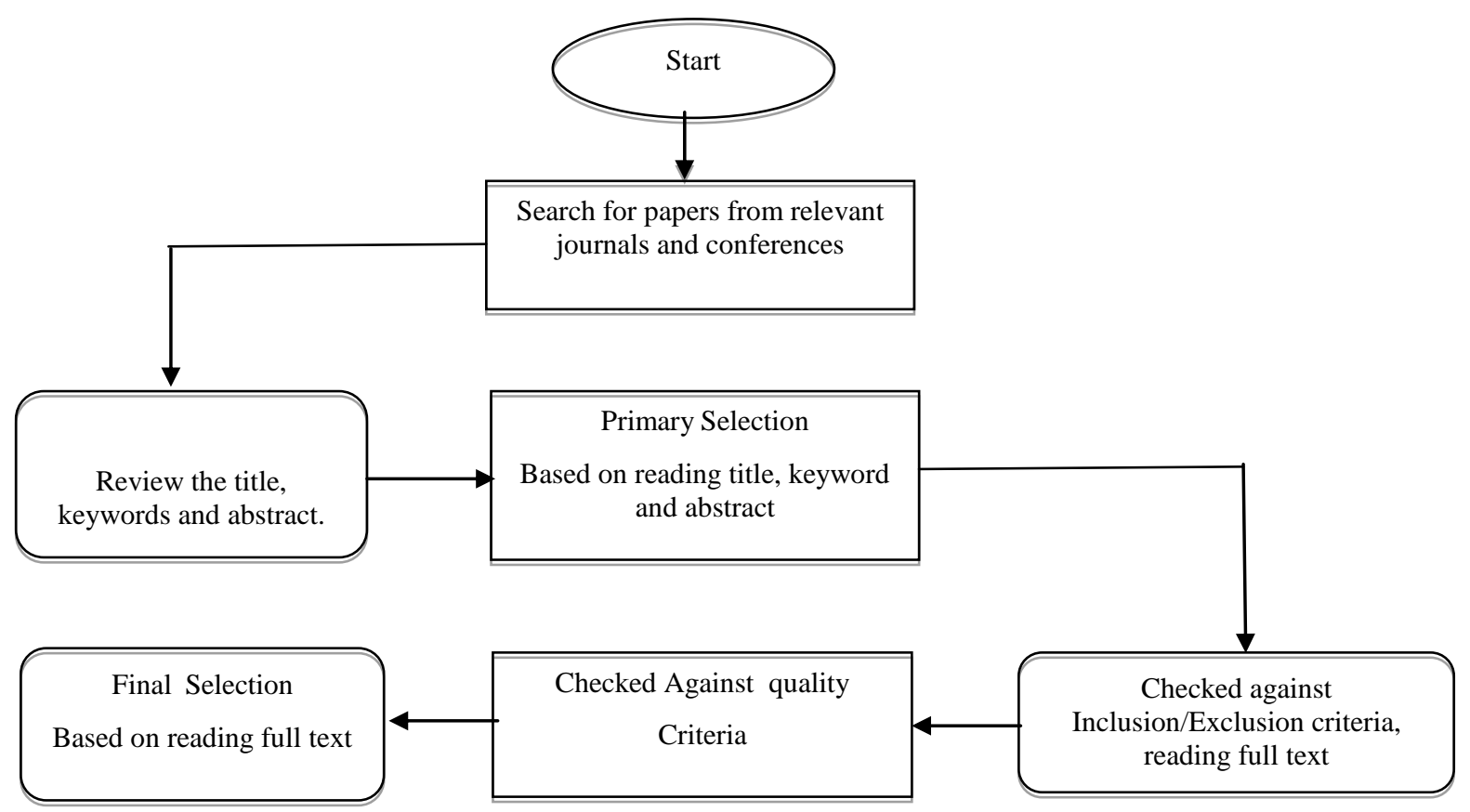

Fig. 2 Publication Selection process

\subsubsection{Exclusion criteria}

On the basis of exclusion criteria, papers that are not to be included will be decided. The following are some exclusion criteria defined for this study.

- Studies which are not related to our Research questions

- $\quad$ Studies that do not discuss RE in GSD

- Studies that do not discuss challenges during RE in GSD.

- Papers belong to GSD but they don't discuss RE

- Papers that discuss RE but don't fit in GSD.

\subsection{Strategy of Data Extraction}

\subsubsection{Primary Study Data}

The data Extracted from publications will contain the following.

- $\quad$ Publication detail (Title, Authors, Reference)

- Data related to research questions

\subsubsection{Data Extraction Process}

One person will do the extraction for review. Secondary person can provide the guidance if he find problems in data extraction.

\subsection{Data synthesis}

The extracted data as a result of SLR will be extracted which give answers of the research questions .The following data will be synthesizes.

- Date of review

- $\quad$ Publication details (Title, Authors, Reference)

- Sample Population

- $\quad$ Company size (small, medium, large)

- Location of the Analysis (continent)

- Publication year

- challenges in requirement implementation in GSD

- Publication Quality Description

Table 2 Study sources and results found

\begin{tabular}{|c|c|c|c|}
\hline Publisher Site & Total Results found & Primary selection & $\begin{array}{c}\text { Final Selected Papers } \\
\text { (Appendix) }\end{array}$ \\
\hline IEEExplore & 360 & 85 & 14 \\
\hline Science Direct & 300 & 85 & 3 \\
\hline ACM & 280 & 40 & 7 \\
\hline Others & 430 & 105 & 2 \\
\hline SpringerLink & 140 & 335 & 30 \\
\hline TOTAL & 1510 & & 3 \\
\hline
\end{tabular}




\section{RESULTS}

In this section, which is organized by the themes and subthemes identified in the research, results related to our research questions are reported. Table 2 lists shows the results after applying and executing SLR protocol. Only 30 papers out of 1510 qualify the inclusion/ exclusion criteria. Finally the duplication was removed by excluded 10 papers from the final list of papers which were repeated across different digital library and the resulted final list of 30 papers are shown in appendix.

\section{CONCLUSION AND FUTURE WORK}

SLR protocol were developed and applied and as result 30 papers are retrieved from different digital libraries. In future, best practices for implementing success factors during successful requirement engineering process in GSD will be retrieved from final list of papers. Questionnaire survey will be conducted for the validation of the identified practices in GSD.

\section{ACKNOWLEDEGMENTS}

We are thankful to the autonomous reviewers of the paper and their valuable comments. We are also thankful to those who appreciated our work and increased our confidence level.

\section{REFERENCES}

[1] M. Yaseen, R. Naseem, Z. Ali, and G. Ullah, 'IDENTIFICATION OF CHALLENGES DURING REQUIREMENTS IMPLEMENTATION IN GLOBAL SOFTWARE DEVELOPMENT: A SYSTEMATIC', vol. 4, no. 1, pp. 23-40, 2019

[2] M. Yaseen, S. Ali, N. Ullah, 'An Improved Framework for Requirement Implementation in the context of Global Software Development: A Systematic Literature Review Protocol', International Journal of Database Theory and Application, 9(6), 161-170, 2016.

[3] Z. Ali, M. Yaseen, and S. Ahmed, 'Effective communication as critical success factor during requirement elicitation in global software development', vol. 8, no. 03, pp. 108-115, 2019.

[4] M. Yaseen, Z. Ali, and M. Humayoun, 'Requirements Management Model (RMM): A Proposed Model for Successful Delivery of Software Projects', Int. J. Comput. Appl., vol. 178, no. 17, pp. 32-36, 2019.

[5] M. . Yaseen, S. . Baseer, S. . Ali, S. U. . Khan, and Abdullahb, 'Requirement Implementation Model (RIM) in the context of global software development. In 2015 International Conference on Information and Communication Technologies (ICICT) (pp. 1-6). IEEE.

[6] M. Yaseen and U. Farooq, 'Requirement Elicitation Model (REM) in the Context of Global Software Development', Glob. J. Comput. Sci. Technol., vol. 1, no. 2, pp. 1-6, 2018.

[7] Z. Ali and M. Yaseen, 'Critical Challenges for Requirement Implementation in Global Software Development: A Systematic Literature Review Protocol with Preliminary Results', vol. 182, no. 48, pp. 17-23, 2019.

[8] M. Yaseen and Z. Ali, 'Success Factors during Requirements Implementation in Global Software Development: A Systematic Literature Review', vol. 8, no. 3, pp. 56-68, 2019.
[9] J. Iqbal, R. Ahmad, M. H. Nizam, M. Nasir, and M. A. Noor, 'Significant Requirements Engineering Practices for Software Development Outsourcing', Proc. 2013 \{22Nd\} Aust. Conf. Softw. Eng., pp. 137-144, 2013.

[10] M. Romero, A. Vizcaino, and M. Piattini, 'Teaching Requirements Elicitation within the Context of Global Software Development', Comput. Sci. (ENC), 2009 Mex. Int. Conf., pp. 232-239, 2009.

[11] J. M. M. Bhat, M. Gupta, and S. N. N. Murthy, 'Overcoming Requirements Engineering Challenges: Lessons from Offshore Outsourcing', IEEE Softw., vol. 23 , no. 5, pp. 38-44, 2006.

[12] M. Geisser, T. Hildenbrand, F. Rothlauf, and C. Atkinson, 'An Evaluation Method for Requirements Engineering Approaches in Distributed Software Development Projects', no. Icsea, 2007.

[13] I. Journal, 'An Improved Framework for Requirement Implementation in the context of Global Software Development : A Systema ....', no. July, 2016.

[14] M. Yaseen, S. Baseer, and S. Sherin, 'Critical Challenges for Requirement Implementation in Context of Global Software Development: A Systematic Literature Review', pp. 120-125, 2015

[15] M. Heindl and S. Biffl, 'Risk management with enhanced tracing of requirements rationale in highly distributed projects', Proc. 2006 Int. Work. Glob. Softw. Dev. Pract. - GSD ’06, p. 20, 2006.

[16] B. Berenbach, 'Impact of Organizational Structure on Distributed Requirements Engineering Processes: Lessons Learned', Int. Work. Glob. Softw. Dev. Pract. GSD 06, pp. 15-19, 2006.

[17] C. Gutwin, R. Penner, and K. Schneider, 'Group Awareness in Distributed Software Development', pp. 72-81, 2004.

[18] C. L. Campbell, B. V. A. N. D. E. Walle, and A. G. S. Development, 'Asynchronous Requirements Engineering: Enhancing Distributed Software Development', pp. 133-136, 2003.

[19] D. Damlan, 'Stakeholders in global requirements engineering: Lessons learned from practice', IEEE Softw., vol. 24, no. 2, pp. 21-27, 2007.

[20] D. Damian, 'An empirical study of requirements engineering in distributed software projects : is distance negotiation more effective ?', vol. 4, no. 04, pp. 149-152, 2001.

[21] D. Requirements, 'Enabling Collaboration in Distributed Requirements Management - ABI INFORM Collection ProQuest', 2006

[22] a. Lopez, J. Nicolas, a. Toval, J. M. Carrillo-de-Gea, and a. Toval, 'Risks and Safeguards for the Requirements Engineering Process in Global Software Development', Glob. Softw. Eng. 2009. ICGSE 2009. Fourth IEEE Int. Conf., no. 1, pp. 394-399, 2009.

[23] M. Geisser, T. Hildenbrand, F. Rothlauf, and C. Atkinson, 'An Evaluation Method for Requirements Engineering Approaches in Distributed Software Development Projects', no. Icsea, 2007.

[24] M. Romero, A. Vizcaino, and M. Piattini, 'Teaching 
Requirements Elicitation within the Context of Global Software Development', Comput. Sci. (ENC), 2009 Mex. Int. Conf., pp. 232-239, 2009.

[25] J. Iqbal, R. Ahmad, M. H. Nizam, M. Nasir, and M. A. Noor, 'Significant Requirements Engineering Practices for Software Development Outsourcing', Proc. 2013 \{22Nd\} Aust. Conf. Softw. Eng., pp. 137-144, 2013.

[26] W. Hussain and T. Clear, 'Spreadsheets as Collaborative Technologies in Global Requirements Change Management', 2014.

[27] C. Ebert, B. K. Murthy, and N. N. Jha, 'Managing Risks in Global Software Engineering: Principles and Practices', 2008 IEEE Int. Conf. Glob. Softw. Eng., pp. 131-140, 2008.

[28] Z. Haag, R. Foley, and J. Newman, 'Software process improvement in geographically distributed software engineering: an initial evaluation', EUROMICRO 97. Proc. 23rd EUROMICRO Conf. New Front. Inf. Technol. (Cat. No.97TB100167), pp. 134-141, 1997.

[29] M. Korkala and P. Abrahamsson, 'Communication in Distributed Agile Development: A Case Study', 33rd EUROMICRO Conf. Softw. Eng. Adv. Appl. (EUROMICRO 2007), no. Seaa, pp. 203-210, 2007.

[30] J. M. M. Bhat, M. Gupta, and S. N. N. Murthy, 'Overcoming Requirements Engineering Challenges: Lessons from Offshore Outsourcing', IEEE Softw., vol. 23, no. 5, pp. 38-44, 2006.

[31] A. Boden, B. Nett, and V. Wulf, 'Coordination practices in distributed software development of small enterprises', Proc. - Int. Conf. Glob. Softw. Eng. ICGSE 2007, no. Icgse, pp. 235-246, 2007.

[32] M. Kauppinen, M. Vartiainen, J. Kontio, S. Kujala, and R. Sulonen, 'Implementing requirements engineering processes throughout organizations: Success factors and challenges', Inf. Softw. Technol., vol. 46, no. 14, pp. 937-953, 2004.

[33] I. Inayat, S. Salwah, S. Marczak, M. Daneva, and S. Shamshirband, 'Computers in Human Behavior A systematic literature review on agile requirements engineering practices and challenges', Comput. Human Behav., 2014.
[34] M. Daneva et al., 'The Journal of Systems and Software Agile requirements prioritization in large-scale outsourced system projects : An empirical study', J. Syst. Softw., vol. 86, no. 5, pp. 1333-1353, 2013.

[35] D. M. Fernández and S. Wagner, 'Naming the pain in requirements engineering: A design for a global family of surveys and first results from Germany', Inf. Softw. Technol., vol. 57, no. 1, pp. 616-643, 2015.

[36] G. N. Aranda, A. Vizcaíno, and M. Piattini, 'A framework to improve communication during the requirements elicitation process in GSD projects', Requir. Eng., vol. 15, no. 4, pp. 397-417, 2010.

[37] A. Lamersdorf and J. Münch, 'A multi-criteria distribution model for global software development projects', J. Brazilian Comput. Soc., vol. 16, no. 2, pp. 97-115, 2010.

[38] T. Leonard, V. Berzins, and M. J. Holden, 'Gathering Requirements from Remote Users', pp. 462-471.

[39] A. Hoffmann and C. Lescher, 'Collaboration and Intercultural Issues on Requirements: Communication, Understanding and Softskills (CIRCUS)', 2009 Collab. Intercult. Issues Requir. Commun. Underst. Softskills, CIRCUS 2009, no. Circus 2009, pp. 1-4, 2009.

[40] G. N. Aranda, A. Vizcaíno, A. Cechich, and M. Piattini, 'Strategies to Minimize Problems in Global Requirements Elicitation RE-GSD Methodology', CLEI Electron. J., vol. 11, no. 1, 2008.

[41] Darja Šmite, 'Requirements Management in Distributed Projects', Knowl. Creat. Diffus. Util., vol. 1, no. 2, pp. 69-76, 2006.

[42] J. Hanisch and B. Corbitt, 'Impediments to requirements engineering during global software development', Eur. J. Inf. Syst., vol. 16, no. 6, pp. 793-805, 2007.

[43] R. Lai and N. Ali, 'A Requirements Management Method for Global Software Development', Humanpub.Org, vol. 1, no. March, pp. 38-58, 2013.

[44] R. E. Gallardo-valencia and S. E. Sim, 'Continuous and Collaborative Validation: A Field Study of Requirements Knowledge in Agile', 2010 